\title{
Impact of antibodies against human leukocyte antigens on long-term outcome in pediatric heart transplant patients: An analysis of the United Network for Organ Sharing database
}

\author{
Joseph W. Rossano, MD, ${ }^{\mathrm{a}}$ David L. S. Morales, MD, ${ }^{\mathrm{b}}$ Farhan Zafar, MD, ${ }^{\mathrm{b}}$ Susan W. Denfield, MD, ${ }^{\mathrm{a}}$ \\ Jeffrey J. Kim, MD, ${ }^{a}$ John L. Jefferies, MD, ${ }^{a}$ and William J. Dreyer, $\mathrm{MD}^{\mathrm{a}}$
}

\begin{abstract}
Objectives: Controversy exists regarding the importance of circulating antibodies as determined by panelreactive antibody screening as a risk factor for graft failure in pediatric patients undergoing heart transplantation. This study sought to determine the association of elevated anti-human leukocyte antibodies with long-term survival in pediatric heart transplant patients.
\end{abstract}

\begin{abstract}
Methods: The United Network for Organ Sharing registry was queried for pediatric patients (aged $<18$ years at listing) with panel-reactive antibody levels obtained before heart transplantation from 1987 through 2004. Survival analysis methods were used to assess the association of elevated panel-reactive antibodies with longterm graft and patient survival.

Results: Panel-reactive antibodies were obtained before transplantation from 3534 patients, median age 4 years (interquartile range 0-12 years). Most, 2711 (77\%), had no detectible panel-reactive antibodies, $436(12 \%)$ had panel-reactive antibodies of $1 \%$ to $10 \%$, and $387(11 \%)$ had panel-reactive antibodies greater than $10 \%$. Patients with panel-reactive antibodies greater than $10 \%$ were more likely to be older $(P=.04)$, have congenital heart disease $(P<.001)$, and have a longer wait list time $(P=.006)$. Patients with panel-reactive antibodies greater than $10 \%$ had significantly worse graft survival and patient survival than did patients with undetectable panelreactive antibodies and panel-reactive antibodies of $1 \%$ to $10 \%(P<.05$ for all). Controlling for confounding variables, elevated panel-reactive antibodies as a continuous variable and panel-reactive antibodies greater than $10 \%$ as a categorical variable were independently associated with decreased graft survival $(P=.04$ and $P=.02$, respectively).
\end{abstract}

Conclusions: Elevated panel-reactive antibodies are independently associated with worse long-term graft survival in pediatric patients undergoing heart transplantation. Further study is needed to determine the optimal management of this high-risk population. (J Thorac Cardiovasc Surg 2010;140:694-9)

Supplemental material is available online.

Heart transplantation in children is routinely performed in many institutions with excellent short- and medium-term

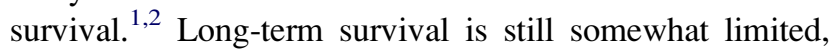
with the median survival after transplantation being 11 to 16 years, depending on the age at transplant. ${ }^{3}$ Many pretransplant factors have limited posttransplant survival,

From the Department of Pediatrics, Lillie Frank Abercrombie Section of Pediatric Cardiology, ${ }^{a}$ and the Michael E. DeBakey Department of Surgery, ${ }^{\mathrm{b}}$ Baylor College of Medicine, Texas Children's Hospital, Houston, Tex.

Disclosures: None.

Received for publication Nov 24, 2009; revisions received March 24, 2010; accepted for publication April 12, 2010; available ahead of print May 31, 2010.

Address for reprints: Joseph W. Rossano, MD, Lillie Frank Abercrombie Section of Pediatric Cardiology, Texas Children's Hospital, 6621 Fannin; MC 19345-C,

Houston, TX 77030 (E-mail: jrossano@bcm.tmc.edu).

$0022-5223 / \$ 36.00$

Copyright (c) 2010 by The American Association for Thoracic Surgery doi:10.1016/j.jtcvs.2010.04.009 including the presence of congenital heart disease, renal insufficiency, and the use of extracorporeal life support. ${ }^{3}$ Additionally, the presence of antibodies directed against human leukocyte antigens (HLA) has been found to be associated with decreased survival in adults. ${ }^{4-7}$

However, there are very limited data on the effect of antiHLA antibodies on survival after pediatric heart transplantation, and the available reports yield conflicting results. Several studies have demonstrated an increased risk of acute rejection among patients with elevated pretransplant antiHLA antibodies, ${ }^{8,9}$ but others have not. ${ }^{10}$ Additionally, there has been some suggestion of decreased survival in this cohort of patients $^{11,12}$; however, this finding has not been consistent. $^{10,13}$ The lack of consistent findings from these studies may be, in part, due to each study being a single center study of relatively small size.

Anti-HLA antibodies are typically measured in pretransplant patients with panel of reactive antibody (PRA) testing. PRA testing involves testing the potential recipient's serum against a panel of HLA antigens, either from lymphocytes or from purified HLA antigens. ${ }^{14}$ The results of PRA testing 


\section{Abbreviations and Acronyms \\ $\mathrm{ECMO}=$ extracorporeal membrane oxygenation \\ HLA = human leukocyte antigen \\ PRA = panel-reactive antibody \\ UNOS $=$ United Network for Organ Sharing \\ STAR $=$ Standard Transplant Analysis and Research \\ $\mathrm{IQR}=$ interquartile range}

are expressed as a percentage of reactivity. Conventionally, a PRA value of more than $10 \%$ is thought to be sensitive for identifying patients at increased risk for adverse outcomes. $^{7,15}$

The United Network for Organ Sharing (UNOS) maintains a database of all solid organ transplants in the United States. Data on pretransplant factors, including PRA levels, are recorded as are outcome data including graft and patient survival. Using the UNOS database, this study aimed to test the hypothesis that elevated pretransplant PRAs would be a risk factor for long-term graft and patient survival in pediatric patients undergoing heart transplantation.

\section{METHODS}

A retrospective analysis was performed on data obtained from the UNOS Standard Transplant Analysis and Research (STAR) files, with additional data provided by UNOS on crossmatch results. Data on heart transplants in the United States from October 1, 1987, with follow-up through May 15, 2008, were included for analysis. PRA data, expressed as a percentage, were available for transplants through June 1,2004. Data were not available during that time period on the specific percentages of PRAs for HLA class I or class II antigens. The database was queried for pediatric patients ( $<18$ years old at time of listing for heart transplantation) who underwent heart transplantation from October 1, 1987, through June 1, 2004, with PRA data before transplantation.

The most recent PRAs before transplantation were used for analysis. Patients were placed into 3 groups, $0 \%, 1 \%$ to $10 \%$, or more than $10 \%$ PRAs. Additional data evaluated included demographic information, indication for transplantation, patient condition at transplantation including the use of inotropic medications or extracorporeal membrane oxygenation (ECMO), donor information, patient survival, and graft survival. The primary end point was graft survival and the secondary end point was patient survival.

\section{Statistical Analysis}

Data from the UNOS STAR files were imported into SPSS, version 16.0 (SPSS Inc, Chicago, Ill), for analysis. Baseline data are reported as means with standard deviations or as percentages, as appropriate. Baseline characteristics were compared among the 3 groups using an analysis of variance with the Bonferroni adjustment for multiple comparisons for continuous variables and a contingency table analysis for categorical variables. Kaplan-Meier survival curves were constructed and log-rank statistics were used to compare overall graft and patient survival among the different PRA groups. Cox proportional hazards models were used to assess the hazard ratios of graft and patient death in patients with elevated PRAs, while controlling for age, gender, diagnosis, year of transplant, inotropic medication at transplant, ECMO at transplant, mechanical ventilation at transplant, donor age, donor weight, donor weight/recipient weight ratio, ischemic time, donor/recipient cytomegalovirus status, and crossmatch status. For the multivariable analysis, values of missing variables were replaced by the most common variable for categorical variables and the median value for continuous variables, as has been done previously. ${ }^{16}$

\section{RESULTS}

A total of 4176 pediatric patients underwent heart transplantation during the study period (1987-2004) with PRA data available for $3534(85 \%)$. Patients with PRA data were older, weighed more, and waited longer for transplantation than patients without PRA data (Table E1) Most, 2711 (77\%), had no detectible PRAs, $436(12 \%)$ had PRAs of $1 \%$ to $10 \%$, and $387(11 \%)$ had PRAs greater than $10 \%$. Patients with PRAs greater than $10 \%$ were more likely to be older, heavier, have congenital heart disease, be on inotropic support at transplant, wait longer for transplant, and have heavier donors $(P<.05$ for all) (Table 1). Evaluation of PRA level by age group demonstrated PRAs greater than $10 \%$ in $10 \%$ of patients aged 0 to 5 years, $12.4 \%$ of patients aged 6 to 13 years, and $11.8 \%$ of patients 13 years of age or older (Figure E1). Patients with PRAs greater than $10 \%$ were also more likely to have a positive crossmatch $(P<.001)($ Table 2).

Long-term graft survival was significantly less in patients with PRAs greater than $10 \%$, median survival 7.1 years (interquartile range [IQR] 5.6-8.5 years), than in patients with PRAs of $1 \%$ to $10 \%$, median survival 9.6 years (IQR 7.8-11.4 years) $(P=.003)$, and patients with undetectable PRAs, median survival 9.8 years (IQR 9.1-10.4 years) $(P=.001)$. Graft survival was not different between patients with undetectable PRAs and those with PRAs of $1 \%$ to $10 \%$ $(P=.75)$. Patient survival was also significantly less in patients with PRAs greater than $10 \%$, median survival 10.3 years (IQR 7.5-13.2 years), than in patients with PRAs of $1 \%$ to $10 \%$, median survival 13.9 (IQR $11.0-16.8$ years) $(P=.04)$, and patients with undetectable PRAs, median survival 12.9 years (IQR 11.6-14.3 years) $(P=.014)$. Patient survival was also not significantly different between patients with PRAs of $1 \%$ to $10 \%$ and those with undetectable PRAs $(P=.94)$ (Figure 1). Among patients with elevated PRAs greater than $10 \%$, survival was similar to that of patients with PRAs of $11 \%$ to $25 \%, 26 \%$ to $50 \%$, and more than $50 \%$ (Figure E2).

A positive crossmatch was found in $320(9 \%)$ patients. Patients with PRAs greater than $10 \%$ and a positive crossmatch had the worst overall graft and patient survival, which was significantly worse than that of patients with PRAs of $10 \%$ or less and a negative crossmatch $(P<.05$ for both $)$ (Figure 2). Patients with PRAs greater than $10 \%$ with a negative crossmatch had worse graft and patient survival than did patients with PRAs of $10 \%$ or less; however, statistical significance was only reached for graft survival in patients with PRAs of $10 \%$ or less and a negative crossmatch. 
TABLE 1. Patient characteristics

\begin{tabular}{|c|c|c|c|c|}
\hline Characteristic & Undetectable PRA $(\mathrm{n}=\mathbf{2 7 1 1})$ & PRA $1 \%-10 \%(n=436)$ & PRA $>10 \%(n=387)$ & $P$ value \\
\hline Age, y & $6.2 \pm 6.3 \dagger$ & $6.6 \pm 6.2$ & $7.1 \pm 6.2 \dagger$ & .043 \\
\hline Weight, $\mathrm{kg}$ & $24.4 \pm 23.9 \dagger$ & $25.4 \pm 24.0$ & $27.2 \pm 23.9 \dagger$ & .043 \\
\hline Female & $1174(43)$ & $178(41)$ & $165(43)$ & .620 \\
\hline Year of transplant & & & & $<.001$ \\
\hline 1987-1992 & $634(79)$ & $110(14)$ & $58(7)$ & \\
\hline 1992-1998 & $1115(77)$ & $196(14)$ & $136(9)$ & \\
\hline 1999-2004 & $962(75)$ & $130(10)$ & $193(15)$ & \\
\hline Diagnosis & & & & $<.001$ \\
\hline Congenital heart disease & $1262(47)$ & $190(44)$ & $200(52)$ & \\
\hline Cardiomyopathy & $1243(46)$ & $199(46)$ & $115(30)$ & \\
\hline Other & $206(8)$ & $47(12)$ & $72(19)$ & \\
\hline Inotropic support* & $875(32)$ & $133(31)$ & $157(41)$ & .003 \\
\hline ECMO* & $131(5)$ & $14(3)$ & $15(4)$ & .258 \\
\hline Mechanical ventilation* & $524(19)$ & $82(19)$ & 75 (19) & .966 \\
\hline Wait list time, mo & $2.3 \pm 4.6 \dagger$ & $2.5 \pm 5.7$ & $3.5 \pm 5.8 \dagger$ & .006 \\
\hline
\end{tabular}

Data are shown as mean $\pm \mathrm{SD}$ or n $(\%) . E C M O$, Extracorporeal membrane oxygenation. $*$ Support at time of transplantation. $\dagger$ Difference between groups $P<.05$.

On multivariable analysis, PRAs greater than $10 \%$ were independently associated with decreased graft survival $(P=.02)$ (Table 3). When we evaluated PRAs as a continuous variable, elevated PRAs were independently associated with decreased graft survival $(P=.04)$. PRAs were not independently associated with patient survival.

\section{DISCUSSION}

This is the first study to demonstrate the incidence and outcome of a large cohort of pediatric patients with elevated pretransplant anti-HLA antibodies. More than $75 \%$ of patients had no detectable PRAs whereas $11 \%$ had PRAs elevated more than $10 \%$. Prior studies from adult patients have determined that risk factors for the development of anti-HLA antibodies include pregnancy, prior exposure to blood products or allograft material, prior transplant, and being on mechanical circulatory support. ${ }^{17}$ Similarly, our findings suggest that pediatric patients with elevated PRAs were more likely to be older and have congenital heart disease, many of whom would have undergone prior cardiac surgery with a risk of exposure to blood products and allograft material.

Additionally, patients undergoing transplantation in the most recent era (1999-2004) had more than twice the incidence of elevated PRAs as patients in the earliest era (1987-1992). This is likely related, in part, to techniques used for PRA measurement, which have changed over time. Complement-dependent cytoxicity assay was likely used virtually exclusively in the early era and has been replaced in most centers by more sensitive techniques such as flow cytometry. ${ }^{17}$ In addition, the complexity of patients at high risk for PRA development, such as patients undergoing transplantation after failed Fontan palliation and patients undergoing retransplantation, has also increased over time. ${ }^{3,18}$ Importantly, year of transplant was controlled for in the multivariable analysis and elevated PRA remained independently associated with worse graft survival.

We did not find an increased risk of elevated PRAs among patients supported with ECMO. Yang and associates ${ }^{19}$ reported previously that $3(31 \%)$ of 13 pediatric transplant patients supported by ECMO had elevated PRAs, but elevated PRAs did not develop in any additional patients receiving ECMO support. Whether the shorter duration of support for pediatric patients on extracorporeal life support as a bridge to transplantation as opposed to adults on left ventricular assist devices accounts for these differences or whether other factors may contribute to this finding merits future inquiry.

Importantly, patients with elevated antibodies as determined by greater than $10 \%$ PRAs had significantly worse overall patient and graft survival. Those with undetectable

TABLE 2. Donor and transplant characteristics

\begin{tabular}{|c|c|c|c|c|}
\hline Characteristic & Undetectable PRA $(n=2711)$ & PRA $1 \%-10 \%(n=436)$ & PRA $>10 \%(n=387)$ & $P$ value \\
\hline Donor age, $y$ & $9.0 \pm 10.9$ & $9.4 \pm 10.9$ & $10.2 \pm 11.0$ & .110 \\
\hline Donor weight, $\mathrm{kg}$ & $29.4 \pm 24.3^{*}$ & $31.5 \pm 25.7$ & $33.0 \pm 25.3^{*}$ & .016 \\
\hline Donor/recipient weight ratio & $1.5 \pm 1.1$ & $1.5 \pm 0.9$ & $1.4 \pm 0.7$ & .225 \\
\hline Ischemic time, $\mathrm{h}$ & $3.6 \pm 1.3$ & $3.5 \pm 1.4$ & $3.5 \pm 1.4$ & .387 \\
\hline Crossmatch positive & $155(6) \dagger$ & $34(8) \ddagger$ & $131(34) \dagger \ddagger$ & $<.001$ \\
\hline Donor CMV positive & $471(17)$ & $72(17)$ & $67(17)$ & .967 \\
\hline Donor CMV positive/recipient CMV negative & $98(4)$ & $10(2)$ & $8(2)$ & .130 \\
\hline
\end{tabular}

Data are shown as mean \pm SD or $\mathrm{n}(\%) . C M V$, Cytomegalovirus. *Difference between groups $P<.05 . \dagger$ and $\ddagger$ indicate difference between groups $P<.001$. 

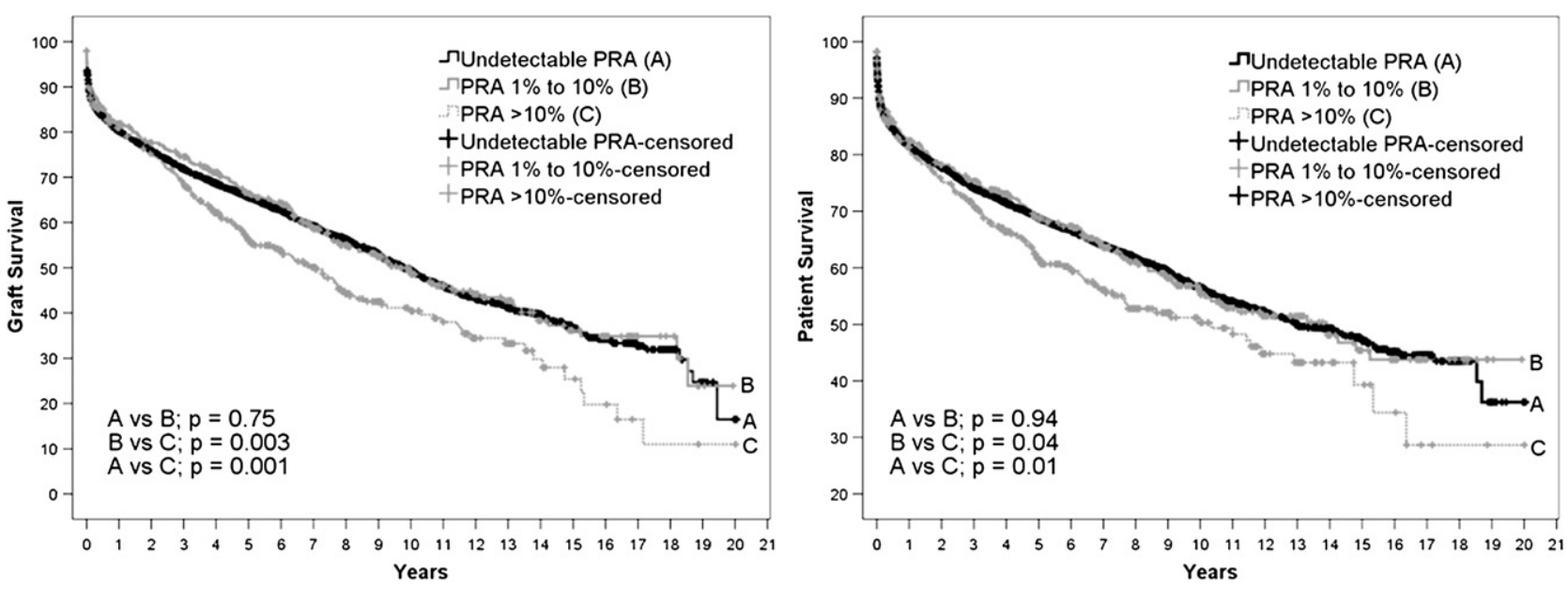

FIGURE 1. Kaplan-Meier survival curve with log-rank statistics for graft and patient survival. PRA, Panel-reactive antibody.

PRAs had a median graft survival over 2.5 years longer than those with PRAs greater than $10 \%$. Additionally, those with PRAs greater than $10 \%$ were significantly more likely to have a positive crossmatch. Although the presence of a positive crossmatch shortened graft and patient survival, patients with elevated PRAs and a negative crossmatch still had worse graft survival than patients without elevated PRAs and a negative crossmatch. It is important to note that the survival in patients with a positive crossmatch and low PRAs was similar to that of patients with a negative crossmatch and high PRAs. These findings are consistent with the concept that patients with elevated PRAs may have shortened graft survival even in the setting of a negative crossmatch.

The association of PRA elevation greater than $10 \%$ and worse graft survival were independent of pretransplant status, year of transplantation, donor factors, crossmatch status, and rejection episodes in the first year after transplant. It is also important to note that the survival of patients with PRAs of $0 \%$ to $10 \%$ was virtually identical to that of patients with undetectable PRAs.
Prior pediatric studies have been limited by the small number of patients with elevated PRA levels, ${ }^{8-13}$ thus limiting the power of these studies to detect important differences in long-term survival. Feingold and colleagues ${ }^{13}$ described 36 patients with PRAs greater than $10 \%$ and found that patients with elevated PRAs had a decreased freedom from coronary allograft vasculopathy, but no statistically significant difference in survival. Holt and coworkers $^{9}$ described the outcomes of 17 patients who underwent transplantation with PRAs greater than $10 \%$. This cohort had a high incidence of rejection $(92 \%)$, a 1-year survival of $85 \%$, and a 3 -year survival of $73 \%$. There was no control group of patients with lower PRAs for this study, but the rates of rejection are higher and survival lower than recent cohorts from the International Society for Heart and Lung Transplantation. ${ }^{3}$ The current study is benefited by including more than 3500 pediatric patients with a long duration of follow-up, which allowed for clinically important differences to be detected. Furthermore, this study strengthens the notion that pediatric patients sensitized
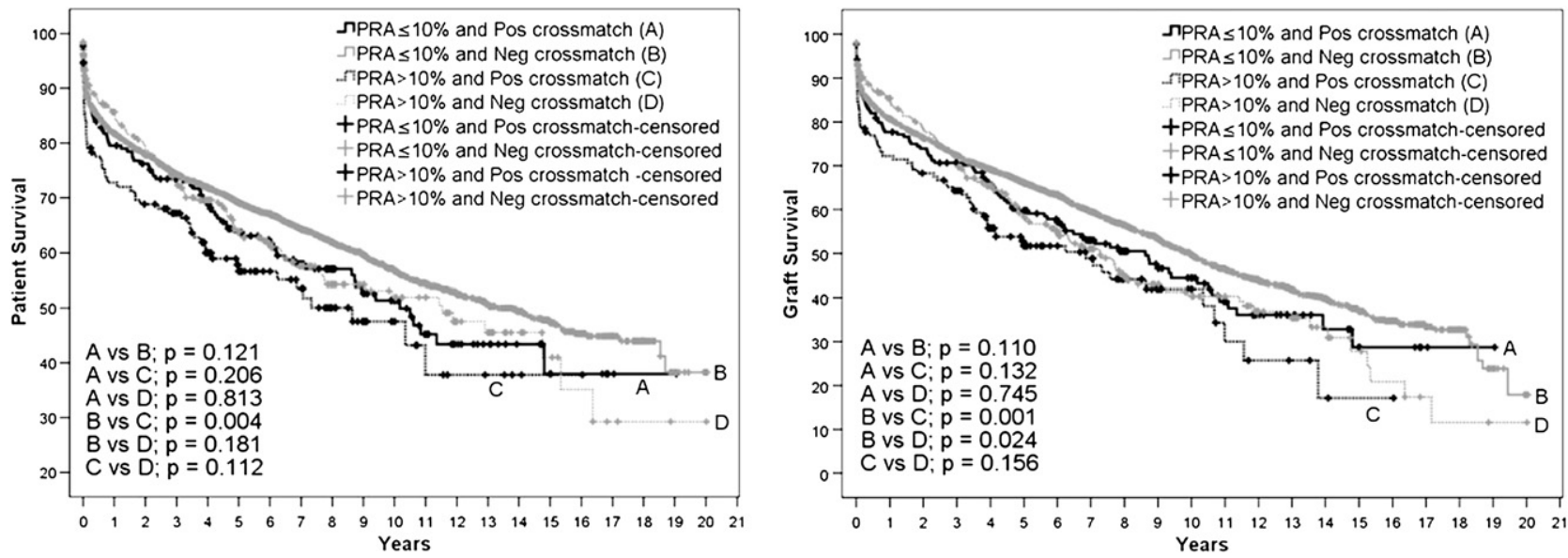

FIGURE 2. Kaplan-Meier survival curve with log-rank statistics for graft and patient survival by crossmatch status. PRA, Panel-reactive antibody. 
TABLE 3. Cox proportional hazard models of graft and patient survival

\begin{tabular}{lcc}
\hline & Hazard ratio $(95 \%$ CI) & $P$ value \\
\hline Graft survival & & \\
PRAs $>10 \%$ & $1.196(1.025-1.396)$ & .02 \\
PRAs, continuous variable & $1.003(1.000-1.006)$ & .04 \\
Patient survival & & \\
PRAs $>10 \%$ & $1.128(0.95-1.339)$ & .17 \\
PRAs, continuous variable & $1.002(0.999-1.005)$ & .27 \\
\hline$C I$, Confidence interval. &
\end{tabular}

with anti-HLA antibodies are a high-risk population at increased risk for earlier graft failure. The precise mechanism by which increased PRAs lead to earlier graft failure are likely multifaceted, including an increased likelihood of a positive crossmatch, ${ }^{20}$ early graft dysfunction, ${ }^{21}$ acute rejection, ${ }^{15,21-24}$ and the earlier development of coronary allograft vasculopathy. ${ }^{13,15}$

\section{Limitations}

This study has several limitations. Data on different techniques for measuring PRAs were not assessed, and thus whether different threshold percentages for PRAs should be used with different techniques cannot be determined. Likewise, the more sensitive tests such as flow cytometry or Luminex ${ }^{24}$ may detect some antibodies that, while present, are not clinically important. ${ }^{25}$ Finally, data were not available on different patient management strategies such as the use of a prospective crossmatch, the use of a virtual crossmatch, or antibody reduction strategies. ${ }^{26}$ Data were also not available on patients who did not survive to transplantation. Although the study is benefited by the large number of patients included and long duration of follow-up data, patient management likely varied considerably among different centers and over time. This variation could affect outcome, and given the limitations of the database, could not be completely controlled for in the multivariable models used.

\section{CONCLUSIONS}

The majority of pediatric heart transplant patients do not have elevated PRAs before transplantation. PRAs greater than $10 \%$ are more common in patients who are older, have congenital heart disease, and are receiving inotropic support at transplant. Elevations in PRAs greater than $10 \%$ are independently associated with worse long-term graft survival in pediatric heart transplant patients. Further study is needed to determine the optimal management of this high-risk population.

We would like to acknowledge the contribution of E. O'Brian Smith, $\mathrm{PhD}$, for his assistance with the statistical analysis. The data reported here have been supplied by the United Network of Organ Sharing on May 20, 2008, as the contractor for the Organ Procurement and Transplantation Network (OPTN). The interpretation and reporting of these data are the responsibility of the authors and in no way should be seen as an official policy of or interpretation by the OPTN or the U.S. Government.

\section{References}

1. Morales DL, Dreyer WJ, Denfield SW, Heinle JS, McKenzie ED, Graves DE, et al. Over two decades of pediatric heart transplantation: how has survival changed? J Thorac Cardiovasc Surg. 2007;133:632-9.

2. Ross M, Kouretas P, Gamberg P, Miller J, Burge M, Reitz B, et al. Ten- and 20-year survivors of pediatric orthotopic heart transplantation. J Heart Lung Transplant. 2006;25:261-70.

3. Kirk R, Edwards LB, Aurora P, Taylor DO, Christie J, Dobbels F, et al. Registry of the International Society for Heart and Lung Transplantation: eleventh official pediatric heart transplantation report-2008. J Heart Lung Transplant. 2008;27: 970-7.

4. Nwakanma LU, Williams JA, Weiss ES, Russell SD, Baumgartner WA, Conte JV. Influence of pretransplant panel-reactive antibody on outcomes in 8,160 heart transplant recipients in recent era. Ann Thorac Surg. 2007;84: 1556-62; discussion 62-63.

5. Przybylowski P, Balogna M, Radovancevic B, Frazier OH, Susskind B, Van Buren $\mathrm{C}$, et al. The role of flow cytometry-detected IgG and IgM anti-donor antibodies in cardiac allograft recipients. Transplantation. 1999;67:258-62.

6. Bishay ES, Cook DJ, El Fettouh H, Starling RC, Young JB, Smedira NG, et al. The impact of HLA sensitization and donor cause of death in heart transplantation. Transplantation. 2000;70:220-2

7. Kobashigawa JA, Sabad A, Drinkwater D, Cogert GA, Moriguchi JD, Kawata N, et al. Pretransplant panel reactive-antibody screens. Are they truly a marker for poor outcome after cardiac transplantation? Circulation. 1996;94(9 Suppl): II294-7.

8. Di Filippo S, Girnita A, Webber SA, Tsao S, Boyle GJ, Miller SA, et al. Impact of ELISA-detected anti-HLA antibodies on pediatric cardiac allograft outcome. Hum Immunol. 2005;66:513-8.

9. Holt DB, Lublin DM, Phelan DL, Boslaugh SE, Gandhi SK, Huddleston CB, et al. Mortality and morbidity in pre-sensitized pediatric heart transplant recipients with a positive donor crossmatch utilizing peri-operative plasmapheresis and cytolytic therapy. J Heart Lung Transplant. 2007;26:876-82.

10. Wright EJ, Fiser WP, Edens RE, Frazier EA, Morrow WR, Imamura M, et al. Cardiac transplant outcomes in pediatric patients with pre-formed anti-human leukocyte antigen antibodies and/or positive retrospective crossmatch. J Heart Lung Transplant. 2007;26:1163-9.

11. Jacobs JP, Quintessenza JA, Boucek RJ, Morell VO, Botero LM, Badhwar V, et al. Pediatric cardiac transplantation in children with high panel reactive antibody. Ann Thorac Surg. 2004;78:1703-9.

12. Pollock-BarZiv SM, den Hollander N, Ngan BY, Kantor P, McCrindle B, Dipchand AI, et al. Pediatric heart transplantation in human leukocyte antigen sensitized patients: evolving management and assessment of intermediate-term outcomes in a high-risk population. Circulation. 2007;116(11 Suppl):I172-8.

13. Feingold B, Bowman P, Zeevi A, Girnita AL, Quivers ES, Miller SA, et al. Survival in allosensitized children after listing for cardiac transplantation. $J$ Heart Lung Transplant. 2007;26:565-71.

14. McKenna RM, Takemoto SK, Terasaki PI. Anti-HLA antibodies after solid organ transplantation. Transplantation. 2000;69:319-26.

15. Kerman RH, Susskind B, Kerman D, Lam M, Gerolami K, Williams J, et al. Comparison of PRA-STAT, sHLA-EIA, and anti-human globulin-panel reactive antibody to identify alloreactivity in pretransplantation sera of heart transplant recipients: correlation to rejection and posttransplantation coronary artery disease. J Heart Lung Transplant. 1998;17:789-94.

16. Patlolla V, Patten RD, Denofrio D, Konstam MA, Krishnamani R. The effect of ventricular assist devices on post-transplant mortality: an analysis of the United Network for Organ Sharing thoracic registry. J Am Coll Cardiol. 2009;53:264-71.

17. Mehra MR, Uber PA, Uber WE, Scott RL, Park MH. Allosensitization in heart transplantation: implications and management strategies. Curr Opin Cardiol. 2003; 18:153-8.

18. Bernstein D, Naftel D, Chin C, Addonizio LJ, Gamberg P, Blume ED, et al. Outcome of listing for cardiac transplantation for failed Fontan: a multi-institutional study. Circulation. 2006;114:273-80

19. Yang J, Schall C, Smith D, Kreuser L, Zamberlan M, King K, et al. HLA sensitization in pediatric pre-transplant cardiac patients supported by mechanical assist devices: the utility of Luminex. J Heart Lung Transplant. 2009;28:123-9. 
20. Smith JD, Danskine AJ, Laylor RM, Rose ML, Yacoub MH. The effect of panel reactive antibodies and the donor specific crossmatch on graft survival after heart and heart-lung transplantation. Transpl Immunol. 1993;1:60-5.

21. Lavee J, Kormos RL, Duquesnoy RJ, Zerbe TR, Armitage JM, Vanek M, et al. Influence of panel-reactive antibody and lymphocytotoxic crossmatch on survival after heart transplantation. J Heart Lung Transplant. 1991;10:921-9; discussion 9-30.

22. Itescu S, Tung TC, Burke EM, Weinberg A, Moazami N, Artrip JH, et al. Preformed IgG antibodies against major histocompatibility complex class II antigens are major risk factors for high-grade cellular rejection in recipients of heart transplantation. Circulation. 1998;98:786-93.

23. DeNofrio D, Rho R, Morales FJ, Kamoun M, Kearns J, Dorozinsky C, et al. Detection of anti-HLA antibody by flow cytometry in patients with a left ventricular assist device is associated with early rejection following heart transplantation. Transplantation. 2000;69:814-8.

24. Tambur AR, Bray RA, Takemoto SK, Mancini M, Costanzo MR, Kobashigawa JA, et al. Flow cytometric detection of HLA-specific antibodies as a predictor of heart allograft rejection. Transplantation. 2000; 70:1055-9.

25. Smith JD, Hamour IM, Banner NR, Rose ML. C4d fixing, Luminex binding antibodies-a new tool for prediction of graft failure after heart transplantation. Am J Transplant. 2007;7:2809-15.

26. Vo AA, Lukovsky M, Toyoda M, Wang J, Reinsmoen NL, Lai CH, et al. Rituximab and intravenous immune globulin for desensitization during renal transplantation. N Engl J Med. 2008;359:242-51. 


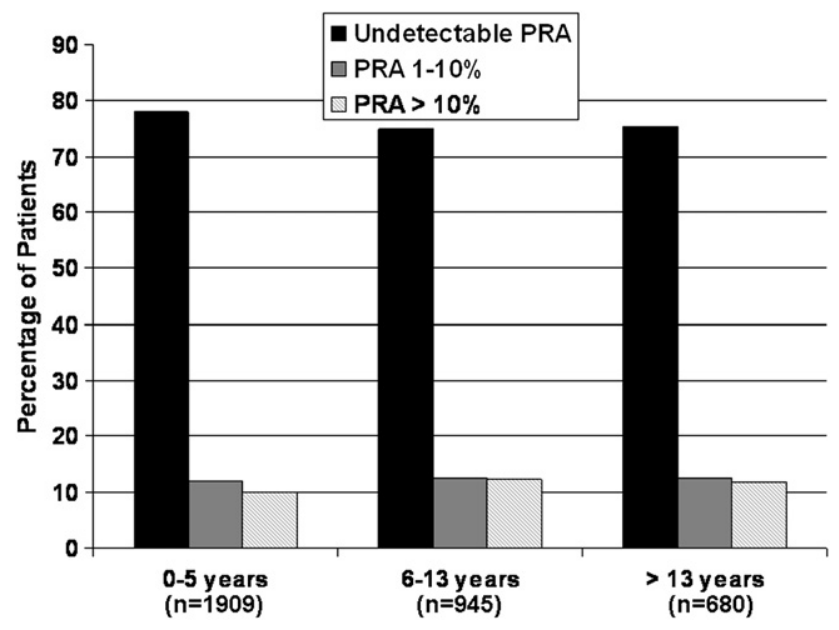

FIGURE E1. Panel-reactive antibody level by age group. $P R A$, Panel-reactive antibody.
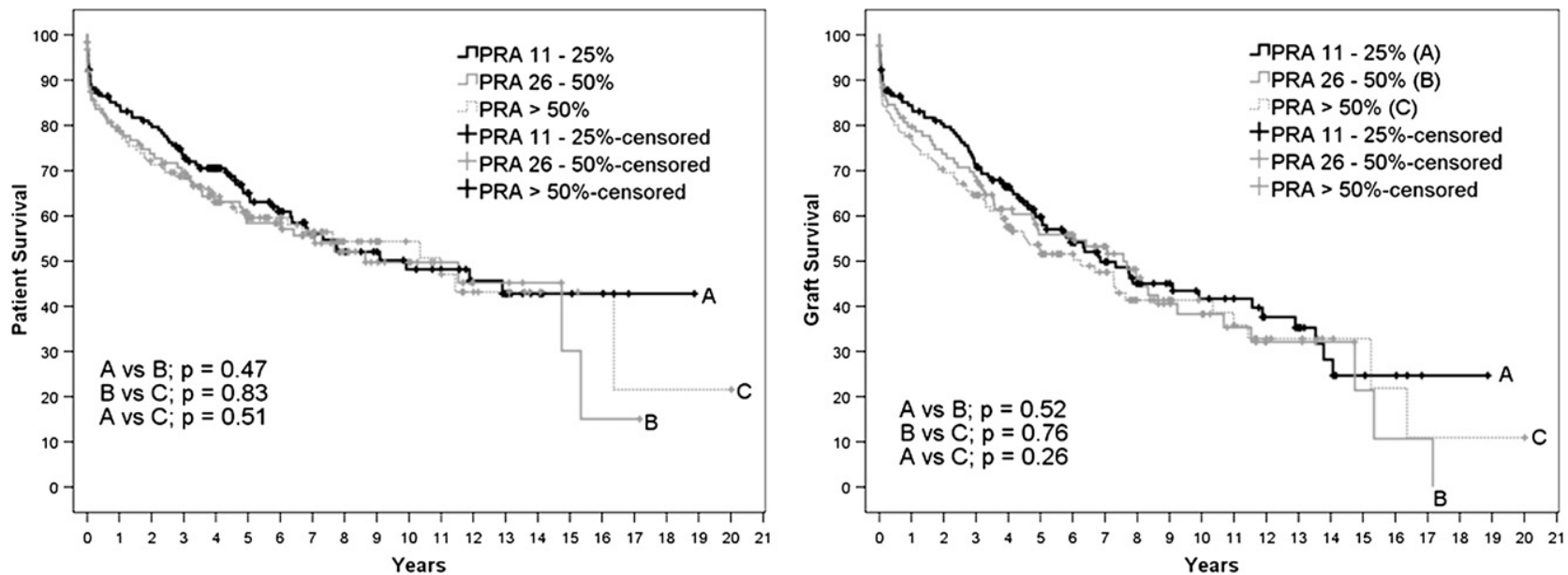

FIGURE E2. Kaplan-Meier survival curve with $\log$-rank statistics for graft and patient survival with panel-reactive antibody levels greater than $10 \%$. PRA, Panel reactive antibody. 
TABLE E1. Characteristics of patients with and without PRA data

\begin{tabular}{|c|c|c|c|}
\hline Characteristic & No PRA data $(n=642)$ & PRA data $(n=3534)$ & $P$ value \\
\hline Age, $y$ & $5.6 \pm 6.2$ & $6.6 \pm 6.2$ & .04 \\
\hline Weight, $\mathrm{kg}$ & $22.0 \pm 22.6$ & $25.2 \pm 23.7$ & .004 \\
\hline Female & $285(44)$ & $1517(43)$ & .49 \\
\hline Year of transplant & & & $<.001$ \\
\hline 1987-1992 & $196(20)$ & $802(80)$ & \\
\hline 1993-1998 & $183(11)$ & $1447(88)$ & \\
\hline 1999-2004 & $263(17)$ & $1285(83)$ & \\
\hline Diagnosis & & & .048 \\
\hline Congenital heart disease & $324(50)$ & $1652(47)$ & \\
\hline Cardiomyopathy & $276(43)$ & $1557(44)$ & \\
\hline Other & $42(7)$ & $325(9)$ & \\
\hline ECMO* & $31(5)$ & $160(5)$ & .74 \\
\hline Mechanical ventilation* & $141(22)$ & $681(19)$ & .11 \\
\hline Wait list time, mo & $2.1 \pm 5.2$ & $2.6 \pm 5.0$ & .02 \\
\hline
\end{tabular}

Data are shown as mean $\pm \mathrm{SD}$ or $\mathrm{n}(\%)$. PRA, Panel-reactive antibody; ECMO, extracorporeal membrane oxygenation. * Support at time of transplantation. 